\title{
A Model of Interacting Partons for Hadronic Structure Functions
}

\author{
G. S. Krishnaswami円 and S. G. Rajeev? \\ Department of Physics and Astronomy, University of Rochester, Rochester, \\ New York 14627
}

\begin{abstract}
We present a model for the structure of baryons in which the valence partons interact through a linear potential. This model can be derived from QCD in the approximation where transverse momenta are ignored. We compare the baryon structure function predicted by our model with a standard global fit to Deep Inelastic Scattering data. The only parameter we can adjust is the fraction of the baryon momentum carried by the valence partons. Our prediction agrees well with data except for small values of the Bjorken scaling variable.

Keywords: Structure Functions; Parton Model; Deep Inelastic Scattering; QCD; Skyrme model; Quantum HadronDynamics.

PACS : 12.39Ki,13.60.-r, 12.39Dc,12.38Aw.
\end{abstract}

\footnotetext{
${ }^{1}$ govind@pas.rochester.edu

${ }^{2}$ rajeev@pas.rochester.edu
} 
The quark-parton model [1] was proposed as a simple explanation of the phenomenon of scaling in Deep Inelastic Scattering. After the discovery of Quantum Chromodynamics (QCD), scaling was understood as a consequence of asymptotic freedom [2]. Moreover, QCD predicts small violations of scaling that can be calculated perturbatively: knowing the parton distribution at one (large enough) energy scale, it is possible to predict them at any other large energy. Although these predictions have been confirmed impressively, there is as yet no theoretical understanding of the initial parton distributions; they are controlled by non-perturbative effects. Accurate knowledge of the parton distributions is essential in order to make predictions about any high energy scattering process in a hadron collider. Also, only after we understand the parton distributions as predicted by the standard model, can we look for deviations which may signal a substructure for quarks. As a result tremendous effort has been expended by several groups of theorists and experimentalists to extract the parton distributions from scattering data at various energy scales 3 , 田, 5.

We will present a model of interacting partons for hadronic structure functions. The partons are assumed to be relativistic particles interacting with each other through a linear potential, which is a sensible idea in the lightcone formalism. Their momenta transverse to the direction of the collision will be ignored. We will solve for the parton wavefunction within the approximation that it factorizes into a product of single particle wavefunctions. This is analogous to the Hartree-Fock approximation of atomic physics. After these approximations, the wavefunction can be determined as the solution of a nonlinear integral equation. This equation can be solved numerically, although we also find a good approximate solution by analytic methods in some special cases.

We can compare with the structure functions extracted by several groups of experimentalists and theorists. Considering that we have at most one parameter to adjust, the agreement is quite good, except for small values of the Bjorken scaling variable $x_{B}$. In this region our model is not expected to be valid: sea quarks and gluons cannot be ignored.

Can we derive this interacting parton model from QCD? In fact we have al- 
ready given such a derivation in previous publications [6]. In the approximation in which transverse momenta are ignored, two of the space-time co-ordinates become irrelevant: QCD is replaced by its dimensional reduction to two dimensions. That such a 'collinear QCD' can describe hadronic structure functions has also been proposed by other scientists [7]. What is unique to our approach is the picture of a baryon as a topological soliton, which remarkably enough, gives a derivation of our interacting parton model from first principles. In previous work, [6] two dimensional QCD was shown in the large $N$ limit to be equivalent to a bilocal field theory whose phase space is an infinite dimensional Grassmannian. The baryon is a topological soliton in this theory, whose energy and structure can be estimated (within a variational approximation) by a nonlinear integral equation. This is precisely the integral equation we will study here. After the equation was derived and studied in this way it became clear that it had a simple interpretation in terms of the parton model. It is this parton model point of view that we will mostly use in this paper.

In this approximation, the dynamical degrees of freedom associated with gluons are ignored: only the longitudinal components of the gluons which can be eliminated in favor of the quarks are kept. In later publications we will study how tranverse gluons will modify the structure of a baryon; in particular derive the gluonic distribution functions.

The stucture functions of a hadron are measured in deep inelastic collisions of a virtual photon ( or weak gauge boson) with a proton or neutron. Since the component of momentum in the direction of collision is much greater than those transverse, it is usually assumed that all the constituents of the hadron (partons) are moving in the same direction. We will choose this direction to be the $x^{1}$-axis. It is also customary to use the null component of momentum $p=p_{0}-p_{1}$ instead of the spatial component as the basic kinematic variable 遖, 3. In terms of the energy $p_{0}$ and null momentum, the mass shell condition for a particle of mass $m$ is

$$
-p^{2}+2 p_{0} p=m^{2}
$$


or equivalently,

$$
p_{0}=\frac{1}{2}\left[p+\frac{m^{2}}{p}\right] .
$$

Note that since $p_{0}=\sqrt{ }\left(p_{1}^{2}+m^{2}\right)>p_{1}$, the null component of momentum $p=p_{0}-p_{1}$ is always positive. 3

Let $\tilde{\psi}(\nu, \alpha, p)$ be the wavefunction of a single parton expressed as a function of the null component of momentum. Here $\alpha=1, \cdots M$ is a discrete quantum number that labels spin and flavor; and $\nu=1, \cdots N$ labels color. Although the value of color in nature is three, we will find it convenient to keep it arbitrary for now. Because of the positivity condition on $p$,

$$
\tilde{\psi}(\nu, \alpha, p)=0, \text { for } p<0
$$

A baryon, which is made up of $N$ such partons, will have a wavefunction $\tilde{\psi}\left(\nu_{1}, \alpha_{1}, p_{1} ; \nu_{2}, \alpha_{2}, p_{2} ; \cdots ; \nu_{N}, \alpha_{N}, p_{N}\right)$. This will vanish unless all the momenta are positive. Since the partons are fermions (after all, they are quarks), the wavefunction must be totally anti-symmetric under permutations of the partons. However, the baryon must be colorless so the color quantum numbers must be completely antisymmetric by themselves:

$\tilde{\psi}\left(\nu_{1}, \alpha_{1}, p_{1} ; \nu_{2}, \alpha_{2}, p_{2} ; \cdots, \nu_{N}, \alpha_{N}, p_{N}\right)=\epsilon_{\nu_{1}, \nu_{2}, \cdots \nu_{N}} \tilde{\psi}\left(\alpha_{1}, p_{1} ; \alpha_{2}, p_{2} ; \cdots \alpha_{N}, p_{N}\right)$

where $\epsilon_{\nu_{1}, \nu_{2}, \cdots \nu_{N}}$ is the Levi-Civita tensor. So we can forget about color and deal with the remaining part of the wavefunction $\tilde{\psi}\left(\alpha_{1}, p_{1} ; \alpha_{2}, p_{2} ; \cdots ; \alpha_{N}, p_{N}\right)$ which is completely symmetric.

The kinetic energy of a system of $N$ partons will be,

$$
\sum_{\alpha_{1} \cdots \alpha_{N}} \int_{0}^{\infty} \sum_{i=1}^{N} \frac{1}{2}\left[p_{i}+\frac{m_{\alpha_{i}}^{2}}{p_{i}}\right]\left|\tilde{\psi}\left(\alpha_{1}, p_{1} ; \cdots ; \alpha_{N}, p_{N}\right)\right|^{2} \frac{d p_{1} \cdots d p_{N}}{(2 \pi)^{N}}
$$

\footnotetext{
${ }^{3}$ The mass shell condition looks a bit unusual beacuse the metric tensor is not diagonal in our choice of variables. If we use the conjugate variables $(u, x)$ to $p_{0}, p, d s^{2}=d u^{2}+2 d u d x$. Here $x$ is the usual spatial co-ordinate and $u=x^{0}-x$ is a null co-ordinate.
} 
If there is a two body potential $g^{2} v(x)$ between the partons, the total potential energy will be 7

$$
\frac{1}{2} g^{2} \sum_{\alpha_{1} \cdots \alpha_{N}} \int_{0}^{\infty} \sum_{i \neq j} v\left(x_{i}-x_{j}\right)\left|\psi\left(\alpha_{1}, x_{1} ; \cdots ; \alpha_{N}, x_{N}\right)\right|^{2} d x_{1} \cdots d x_{N}
$$

Here,

$$
\psi\left(\alpha_{1}, x_{1} ; \cdots \alpha_{1}, x_{N}\right)=\int_{0}^{\infty} \tilde{\psi}\left(\alpha_{1}, p_{1} ; \cdots \alpha_{N}, p_{N}\right) e^{i \sum_{j} p_{j} x_{j}} \frac{d p_{1} \cdots d p_{N}}{(2 \pi)^{N}}
$$

is the wavefunction in position space.

In addition to the kinetic and potential energies, there could be a term in the hamiltonian describing the self-energy of the partons. Due to Lorentz invariance, such a term can only be a (finite) renormalization of $m^{2}$; i.e., it is of the form

$$
c \sum_{\alpha_{1} \cdots \alpha_{N}} \int_{0}^{\infty} \sum_{i=1}^{N} \frac{g^{2}}{2 \pi p_{i}}\left|\tilde{\psi}\left(\alpha_{1}, p_{1} ; \cdots ; \alpha_{N}, p_{N}\right)\right|^{2} \frac{d p_{1} \cdots d p_{N}}{(2 \pi)^{N}}
$$

for some constant $c$.

Thus, the ground state wavefunction is determined by minimizing the total energy

$$
\begin{aligned}
\mathcal{E}_{N}(\tilde{\psi})= & \sum_{\alpha_{1} \cdots \alpha_{N}} \int_{0}^{\infty} \sum_{i=1}^{N} \frac{1}{2}\left[p_{i}+\frac{\mu_{\alpha_{i}}^{2}}{p_{i}}\right]\left|\tilde{\psi}\left(\alpha_{1}, p_{1} ; \cdots \alpha_{N}, p_{N}\right)\right|^{2} \frac{d p_{1} \cdots d p_{N}}{(2 \pi)^{N}} \\
& +\frac{1}{2} g^{2} \sum_{\alpha_{1} \cdots \alpha_{N}} \int_{0}^{\infty} \sum_{i \neq j} v\left(x_{i}-x_{j}\right)\left|\psi\left(\alpha_{1}, x_{1} ; \cdots \alpha_{N}, x_{N}\right)\right|^{2} d x_{1} \cdots d x_{N}
\end{aligned}
$$

Here $\mu_{\alpha}^{2}=m^{2}+c \frac{g^{2}}{\pi}$ is the renormalized mass of the parton.

The success of the simple parton model and its QCD inspired variants shows that it is a good approximation to describe the system in terms of particle distributions that depend only on the momentum of a single parton. In other words correlations between the partons can be ignored, except in that the total momentum of the partons must be fixed. Thus it should be a good approximation

\footnotetext{
${ }^{4}$ A multiplicative factor has been chosen such that the coupling constant $g$ has units of mass.
} 
to assume that (in the ground state), the baryon wavefunction has the form :

$$
\tilde{\psi}\left(\alpha_{1}, p_{1} ; \cdots \alpha_{N}, p_{N}\right)=2 \pi \delta\left(P-\sum_{i} p_{i}\right) \prod_{i=1}^{N} \tilde{\psi}\left(\alpha_{i}, p_{i}\right) .
$$

Here, $P$ is the momentum of the baryon. Since each of the parton momenta $p_{i}$ are positive, we see that

$$
\tilde{\psi}(p)=0 \text { unless } 0 \leq p \leq P .
$$

This ansatz is analogous to the Hartree approximation of atomic physics, which works well even when the number of electrons is small as in the Helium atom. In any case we can regard our product as a variational ansatz for the ground state of the baryon. We will normalize the single parton wavefunction to have length one:

$$
\|\tilde{\psi}\|^{2}=\sum_{\alpha=1}^{M} \int_{0}^{P}|\tilde{\psi}(\alpha, p)|^{2} \frac{d p}{2 \pi}=1 .
$$

Moreover it must satisfy the sum rule on momentum: the total momentum of all the partons must equal the momentum of the baryon:

$$
N \int_{0}^{P} p|\tilde{\psi}(p)|^{2} \frac{d p}{2 \pi}=P .
$$

In fact the valence partons may not carry all the momentum of the baryon. (The structure functions extracted from data show that only about half the momentum of the baryon is carried by the valence partons.) We can allow for this by replacing the momentum sum rule above by

$$
N \int_{0}^{P} p|\tilde{\psi}(p)|^{2} \frac{d p}{2 \pi}=f P
$$

where $f$ is the fraction of the baryon momentum carried by all the valence partons. We will see that this fraction $f$ is the only parameter on which the sturcture functions will depend. 
The energy per parton becomes now

$$
\begin{aligned}
E= & \sum_{\alpha} \int_{0}^{P} \frac{1}{2}\left[p+\frac{\mu_{\alpha}^{2}}{p}\right]|\tilde{\psi}(\alpha, p)|^{2} \frac{d p}{2 \pi}+ \\
& \frac{1}{2} \tilde{g}^{2} \int_{-\infty}^{\infty} v(x-y) \sum_{\alpha}|\psi(\alpha, x)|^{2} \sum_{\beta}|\psi(\beta, y)|^{2} d x d y
\end{aligned}
$$

where, $\tilde{g}^{2}=g^{2} N$ and $\psi(\alpha, x)=\int_{0}^{P} \tilde{\psi}(p) e^{i p x} \frac{d p}{2 \pi}$.

What potential $v(x)$ should we use? It is known that the potential between quarks is, to a good approximation, linear. Moreover the collinear approximation to QCD mentioned above predicts a linear potential. Hence we will choose

$$
v(x)=\frac{1}{2}|x| .
$$

A linearly increasing potential can lead to an infrared divergence in the energy of a wavefunction, even one that is decaying at infinity. For example if $\psi(\alpha, x) \sim \frac{1}{x}$ for large $x$ the potential energy term will diverge logarithmically. This is where a proper choice of the self-energy term mentioned above comes in. If we choose the constant $c$ in the self-energy such that

$$
\mu_{\alpha}^{2}=m_{\alpha}^{2}-\frac{\tilde{g}^{2}}{\pi}
$$

this Infrared divergence dissappears. This is indeed the value of the self-energy term predicted by ( the large $N$ limit of ) two dimensional QCD.

The wavefunction of the partons in a baryon is now determined by minimizing the above energy function subject to the constraints on the wavefunction

$$
\tilde{\psi}(p)=0 \text { for } p<0 \text { andfor } p>P \text { and }\|\tilde{\psi}\|^{2}=1 \text {. }
$$

The minimization of the above energy is equivalent to the solution of a system of nonlinear integral equations. The condition that the wavefunction vanish outside of the interval $[0, P]$ in momentum space is a subtle constraint on the position space wavefunction: $\psi(\alpha, x)$ must be the boundary value of an 
entire function which grows at most like $e^{P|x|}$ at infinity. Since such a condition is clearly difficult to impose numerically, we work in momentum space. Variation of the energy with respect to $\tilde{\psi}$ gives

$$
\left[\frac{1}{2}\left(p+\frac{\mu_{\alpha}^{2}}{p}\right)-\lambda\right] \tilde{\psi}(\alpha, p)+\tilde{g}^{2} \mathcal{P} \int_{0}^{P} \tilde{V}(p-q) \tilde{\psi}(\alpha, q) \frac{d q}{2 \pi}=0
$$

where

$$
\tilde{V}(p)=-\frac{1}{p^{2}} \sum_{\alpha} \int_{0}^{P} \tilde{\psi}^{*}(\alpha, p+q) \tilde{\psi}(\alpha, q) \frac{d q}{2 \pi} .
$$

Here, $\lambda$ is the Lagrange multiplier that enforces the constraint $\|\tilde{\psi}\|^{2}=1$. (Since the function we are minimizing is quartic in $\tilde{\psi}$ this is not the same as the minimum energy $E$.)

The second equation is just the momentum space version of the equation for the mean potential due to all the partons:

$$
V^{\prime \prime}(x)=|\psi(x)|^{2} .
$$

Note that the Fourier transform $\tilde{V}(p)=\int_{-\infty}^{\infty} V(x) e^{-i p x} d x$ of the mean potential is singular at the origin:

$$
\tilde{V}(p) \sim-\frac{1}{p^{2}} \text { for } p \rightarrow 0 .
$$

Hence the integrand in the equation for $\tilde{\psi}(\alpha, p)$ is singular. We must define it to be a finite part integral in the sense of Hadamard [8]:

$\mathcal{P} \int_{-\infty}^{\infty} \tilde{V}(p-q) \tilde{\psi}(\alpha, q) \frac{d q}{2 \pi}=\int_{0}^{\infty}[\tilde{\psi}(\alpha, p+q)+\tilde{\psi}(\alpha, p-q)-2 \tilde{\psi}(\alpha, p)] \tilde{V}(q) \frac{d q}{2 \pi}$.

By studying the behavior of the equation in the neighborhood of the singular point $p=0$, with the ansatz $\tilde{\psi}(p) \sim p^{\nu}$, we get the following formula relating the exponent $\nu$ and $m^{2}$ :

$$
\frac{\pi m^{2}}{\tilde{g}^{2}}=1+\int_{0}^{1} \frac{d y}{y^{2}}\left[(1+y)^{\nu}+(1-y)^{\nu}-2\right]+\int_{1}^{\infty} \frac{d y}{y^{2}}\left[(1+y)^{\nu}-2\right] .
$$

Thus, at the critical point $m=0$, we have $\nu=0$ : the critical wavefunction tends to a constant as $p \rightarrow 0$. If $m^{2}>0$, the wavefunction vanishes like a power: $\nu>0$. 
This nonlinear integral equation was derived previously [6] from the large $N$-limit of two dimensional QCD. The 'master field' of this limit of 2DQCD is a hermitean matrix $\tilde{M}(\alpha, p ; \beta, q)$ satisfying the nonlinear constraint

$$
\tilde{M}(\alpha, p ; \beta, q)[\operatorname{sgn}(p)+\operatorname{sgn}(q)]+\sum_{\gamma} \int \frac{d r}{2 \pi} \tilde{M}(\alpha, p ; \gamma, r) \tilde{M}(\gamma, r ; \beta, q)=0 .
$$

The set of solutions of this condition is an infinite dimensional Grassmannian manifold, which has connected components labelled by an integer: the 'virtual rank', $-\frac{1}{2} \sum_{\alpha} \int \tilde{M}(\alpha, p ; \alpha, p) \frac{d p}{2 \pi}$. This integer has the physical meaning of baryon number. The baryon is thus a topological soliton and is described by a configuration of minimal energy in the sector of virtual rank one.

Thus we have here a realization of Skyrme's idea that the baryon is a topological soliton 9]. The energy of the configuration $\tilde{M}(p, q)$ is given by

$$
\mathcal{E}(\tilde{M})=\sum_{\alpha} \int \frac{1}{2}\left(p+\frac{\mu_{\alpha}^{2}}{p}\right) \tilde{M}(\alpha, p ; \alpha, p) \frac{d p}{2 \pi}+\tilde{g}^{2} \sum_{\alpha, \beta} \int|M(\alpha, x ; \beta, y)|^{2} v(x-y) d x d y .
$$

Here,

$$
M(\alpha, x ; \beta, y)=\int \tilde{M}(\alpha, p ; \beta, q) e^{i p x-i q y} \frac{d p d q}{(2 \pi)^{2}}
$$

The parameters $\mu_{\alpha}^{2}$ and $\tilde{g}$ are related to those of 2DQCD:

$$
\mu_{\alpha}^{2}=m_{\alpha}^{2}-\frac{\tilde{g}^{2}}{\pi}, \quad \tilde{g}^{2}=g^{2} N
$$

where $m$ is the current quark mass and $g$ the gauge coupling constant. Note that in the limit of chiral symmetry, $m_{\alpha}=0$, (when the lightest meson is massless) and the value of $\mu_{\alpha}^{2}$ is actually negative:

$$
\mu_{\alpha}^{2}=-\frac{\tilde{g}^{2}}{\pi}
$$

This is the value we will mostly study.

In addition to topological soliton solutions describing the baryon the above equation also describes small fluctuations from the vacuum. The corresponding particles are the mesons. If we assume that $\tilde{M}(p, q)$ is infinitesimally small, its equations of motion will reduce to a linear integral equation. This equation 
was first derived by 't Hooft by a masterful use of diagrammatic methods [10]. Witten [11] suggested later that the baryon can be described by a Hartree-Fock aproximation in the large $N$ limit of QCD. He carried out this idea in a nonrelativistic context. Our model may be thought of a relativistic implementation of this idea of Witten.

Now if we make the variational ansatz that $\tilde{M}(\alpha, p ; \beta, q)=-2 \tilde{\psi}(\alpha, p) \tilde{\psi}^{*}(\beta, q)$ is separable, the quadratic constraint on $\tilde{M}$ becomes the condition that

$$
\tilde{\psi}(\alpha, p)=0 \text { for } p<0 .
$$

Moreover, the energy of the soliton reduces to just the formula we obtained within the parton model! (The momentum sum rule is the the analogue of the WKB quantization condition in this theory.) This is how we can derive the parton model from collinear QCD. It is remarkable that the topological soliton model, which at first looks so different from the parton model, leads to exactly the same equation for the hadron structure function.

The variational approximation that $\tilde{M}$ be separable corresponds in the parton model to ignoring the 'sea quarks'. This seems reasonable only for values of the Bjorken parameter $x_{B}$ that are not too small. In general we should get better answers by directly minimizing the energy of the soliton model without the further approximation of the separable ansatz. We are currently studying this issue.

Let us return to solving the integral equation. It cannot be solved analytically, so we will resort to a numerical method. ( We can get approximate analytical solutions in some special cases which agree well with the numerical solution.) For simplicity, we will from now on put all the parton masses equal to each other. It is not difficult to modify our solutions to include unequal masses. Also, it is sufficient to find a solution that is non-zero only for one value of the spin-flavor index $\alpha$ :

$$
\tilde{\psi}(\alpha, p)=\delta_{\alpha, 1} \tilde{\psi}(p)
$$

This breaks the $U(M)$ invariance of our model spontaneously. This symme- 
try can be restored later by the collective variable method as in the theory of solitons, but we will not address this issue here.

Let us first describe our approximate analytic solution for the special case $\mu=0$ and large $N$. We are mostly interested in the case $\mu^{2}=-\frac{\tilde{g}^{2}}{\pi}$ but this approximate solution will help to validate our numerical procedure. It is reasonable to expect that most of the contribution to the integral

$$
\int_{0}^{\infty}[\tilde{\psi}(p+q)+\tilde{\psi}(p-q)-2 \tilde{\psi}(p)] \tilde{V}(q) \frac{d q}{2 \pi}
$$

will come from the neighborhood of the singularity near $q=0$ :

$$
\int_{0}^{\infty}[\tilde{\psi}(p+q)+\tilde{\psi}(p-q)-2 \tilde{\psi}(p)] \tilde{V}(q) \frac{d q}{2 \pi} \sim \tilde{\psi}^{\prime \prime}(p) \int_{0}^{\infty} q^{2} \tilde{V}(q) \frac{d q}{2 \pi} .
$$

Set

$$
a=-\int_{0}^{\infty} q^{2} \tilde{V}(q) \frac{d q}{2 \pi}
$$

Using the fact that the ground state wavefnction is real, we can show that

$$
a=\frac{1}{2}\left|\int_{0}^{\infty} \tilde{\psi}(p) \frac{d p}{2 \pi}\right|^{2} .
$$

Thus we get a differential equation:

$$
-\tilde{g}^{2} a \tilde{\psi}^{\prime \prime}(p)+\frac{1}{2}\left[p+\frac{\mu^{2}}{p}-2 \lambda\right] \tilde{\psi}(p)=0 .
$$

This can be thought of as a Schrödinger equation with a linear potential plus a Coulomb potential; such an equation has been used to study heavy quark-antiquark bound states [12]. But the physical origin of our equation is completely different: we get this equation in momentum space not position space. Also, our quarks are not assumed to be heavy and we are studying a baryon not a meson.

In the special case $\mu^{2}=0$, the solution is a linear combination of Airy functions. But it is awkward to work with this linear combination. If we make the further approximation that $N$ is large, our momentum sum rule shows that 
$P$ is large as well. Then the boundary condition on the wavefunction is that it must tend to zero at infinity: in other words

$$
\tilde{\psi}(p)=C \operatorname{Ai}\left(\frac{p-2 \lambda}{\left(2 a \tilde{g}^{2}\right)^{\frac{1}{3}}}\right)
$$

The eigenvalue $\lambda$ is determined by the condition that the solution vanish at the origin:

$$
\lambda=-\frac{1}{2} \xi_{1}\left(2 a \tilde{g}^{2}\right)^{\frac{1}{3}} .
$$

Here, $\xi_{1} \sim-2.33811$ is the root of the Airy function closest to the origin. $C$ is determined by the normalization condition.

The constant $a$ is determined by putting this solution back into the definition of $a$. We get,

$2 a=\tilde{g} \frac{1}{(2 \pi)^{\frac{3}{2}}} \frac{\left|\int_{\xi_{1}}^{\infty} \mathrm{Ai}(\xi) d \xi\right|^{3}}{\left[\int_{\xi_{1}}^{\infty} \mathrm{Ai}^{2}(\xi) d \xi\right]^{\frac{3}{2}}}, \quad \lambda=\frac{\left|\xi_{1}\right|}{2 \sqrt{ }(2 \pi)} \frac{\int_{\xi_{1}}^{\infty} \mathrm{Ai}(\xi) d \xi}{\left[\int_{\xi_{1}}^{\infty} \mathrm{Ai}^{2}(\xi) d \xi\right]^{\frac{1}{2}}} \tilde{g} \sim 0.847589 \tilde{g}$.

Moreover

$$
\tilde{\psi}(p)=C \operatorname{Ai}\left(\xi_{1}\left(\frac{p}{\tilde{g} \lambda}-1\right)\right)
$$

This analytic approximation agrees well with the numerical solution (described below) for $\mu^{2}=0$ and large $N$. This confirms the validity of our numerical method.

Now let us discuss the numerical solution of this problem. This is not straightforward since the kernel of the integral equation is singular. We need a reliable method of numerical quadrature for integrals such as

$$
\mathcal{P} \int_{0}^{P} f(p, q) \rho(q) d q
$$

when the weight function $\rho(q)$ has a singularity at $q=0$ like $\frac{1}{q^{2}}$. We need to subdivide the interval $[0, P]=\cup_{r=1}^{n}\left[b_{r}, b_{r+1}\right]$ into subintervals. Within each 
subinterval we choose a set of points $q_{j r}, j=1, \cdots \nu_{r}$. We approximate the integral by a sum

$$
\int_{0}^{P} f(p, q) \rho(q) d q=\sum_{j r} w_{j r} f\left(q_{j r}\right)
$$

The weights $w_{j r}$ are determined by the condition that within each subinterval $\left[b_{r}, b_{r+1}\right]$, the integral of a polynomial of order $\nu_{r}-1$ is reproduced exactly:

$$
\mathcal{P} \int_{b_{r}}^{b_{r+1}} q^{k} \rho(q) d q=\sum_{j=1}^{\nu_{r}} w_{j r} q_{j r}^{k} .
$$

This is just the usual method of numerical quadrature except that the integral on the left hand side is singular for $b_{r}=0$ and $k=0,1$. In these cases we can evaluate the l.h.s analytically as the finite part in the sense of Hadamard. (The main difference from the usual situation is that the moments on the l.h.s of the above equation are not all positive.) The weights are then determined by solving the above system of linear equations.

Given an approximate mean potential $\tilde{V}_{s}(p)$ we can convert the linear integral equation

$$
\frac{1}{2}\left[p+\frac{\mu^{2}}{p}-2 \lambda_{s}\right] \tilde{\psi}_{s+1}(p)+\tilde{g}^{2} \mathcal{P} \int_{0}^{\infty} \tilde{V}_{s}(p-q) \tilde{\psi}_{s+1}(q) \frac{d q}{2 \pi}=0
$$

into a matrix eigenvalue problem by the above method of quadrature. We use the ground state eigenfunction so determined to calculate numerically the next approximation $\tilde{V}_{s+1}$ for the mean potential. This process is iterated until the solution converges. Having determined the wavefunction, we must impose the momentum sum rule to determine $\tilde{g}$.

We chose $n=50$ intervals each containing $\nu_{r}=6$ points for our quadrature. The resulting matrix was diagonalized using Mathematica. As a starting mean potential we used

$$
\tilde{V}_{0}(q)=-\frac{e^{-\left(\frac{q}{g}\right)^{\frac{3}{2}}}}{q^{2}} .
$$

The procedure converges in about 10 iterations. We find that due to the errors in the discretization of the problem, the critical value of $\mu^{2}$ (the value at which 
the wavefunction goes to a constant at $p=0)$ is a bit lower $\left(-1.3 \frac{\tilde{g}^{2}}{P i}\right)$ than the theoretical value of $-\frac{\tilde{g}^{2}}{\pi}$.

Now we turn to the question of the comparison of our model with data from Deep Inelastic Scattering. It is customary to describe the parton distributions as a function of the Bjorken scaling variable $0 \leq x_{B} \leq 1$ which is the fraction of the null component of momentum carried by each parton. This means we will rescale momenta to the dimensionless variable $p=x_{B} P$. The probability density of a parton carrying a fraction $x_{B}$ of the momentum is then

$$
\phi\left(x_{B}\right)=\frac{P}{2 \pi}\left|\tilde{\psi}\left(x_{B} P\right)\right|^{2} .
$$

(The factor of $\frac{1}{2 \pi}$ is needed because $\phi\left(x_{B}\right)$ is traditionally normalized to one with the measure $d x_{B}$ rather than $\frac{d x_{B}}{2 \pi}$.)

It is important to note that the only dimensional parameter in our theory, $\tilde{g}$, cancels out of the formula for $\phi\left(x_{B}\right)$ : it only serves to set the scale of momentum and when the wavefunction is expressed in terms of the dimensionless variable $x_{B}$ it cancels out. We have set $\mu^{2}$ to the critical value (within numerical errors), which is the value corresponding to chiral symmetry; i.e., zero current quark mass. The number of colors we fix at $N=3$. Thus the only free parameter in our theory is the fraction $f$ of the baryon momentum carried by the valence partons. The parameters $N$ and $f$ appear in the combination $N_{\text {eff }}=\frac{N}{f}$.

We have ignored the ispospin of the quarks in the above discussion. We should therefore compare our structure functions with the isoscalar combination of the valence quark distributions of a baryon, $\phi\left(x_{B}\right)$. It is not difficult to take into account of isospin effects.

The parton distributions have been extracted from scattering data by several groups of physicists [3, 4, 5]. In the accompanying figures we plot our wavefunctions for a few values of $f$ to those extracted from data by the MRST collaboration. We agree remarkably well with experiment except for small values of $x_{B}$. The agreement is best when the fraction of the baryon momentum carried by the valence partons is about $f=0.6$.

Our model does not predict the observed behavior of the parton distributions 
for small $x_{B}$ : our probability distribution tends to a constant for small $x_{B}$ although due to numerical errors that is not evident in the plots. The observed distributions have an integrable singularity there: roughly speaking, $\phi\left(x_{B}\right) \sim$ $x_{B}^{-0.5}$ for small $x_{B}$. The approximations we made clearly break down in the small $x_{B}$ region: sea quarks can no longer be ignored, indeed even gluons need to be considered. We will study these effects in future publications.

\section{Acknowledgement}

This research was supported by the Department of Energy through the grant

DE-FG02-91ER40685 and by the Summer Reach program of the University of Rochester. 


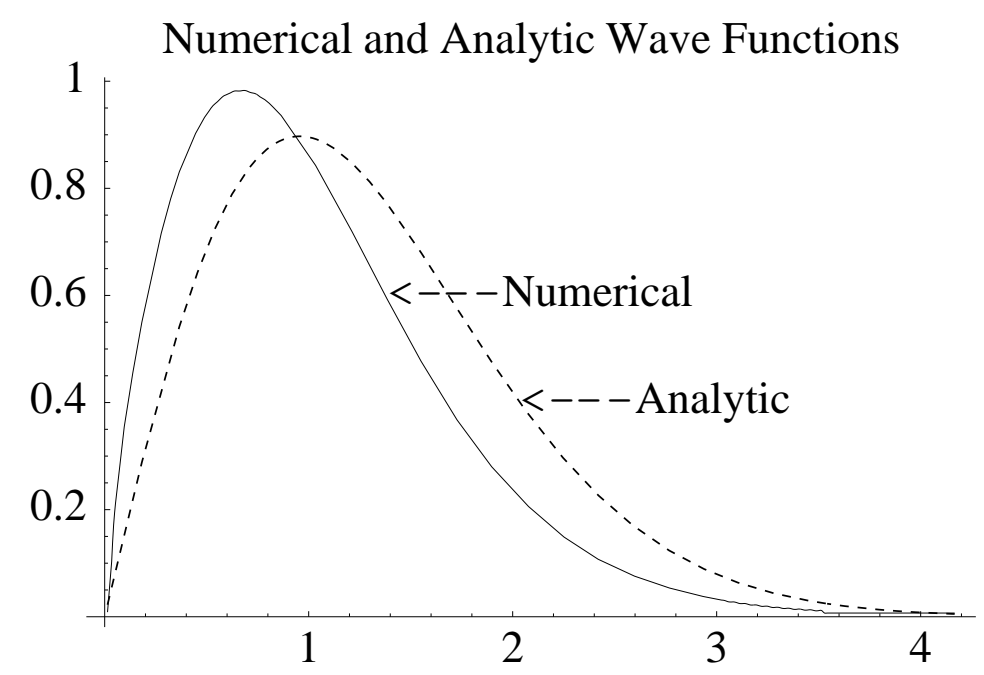

The numerical solution for $\mu^{2}=0, N=5$ compared against the approximate analytical solution. The horizontal axis is $\frac{p}{\tilde{g}}$

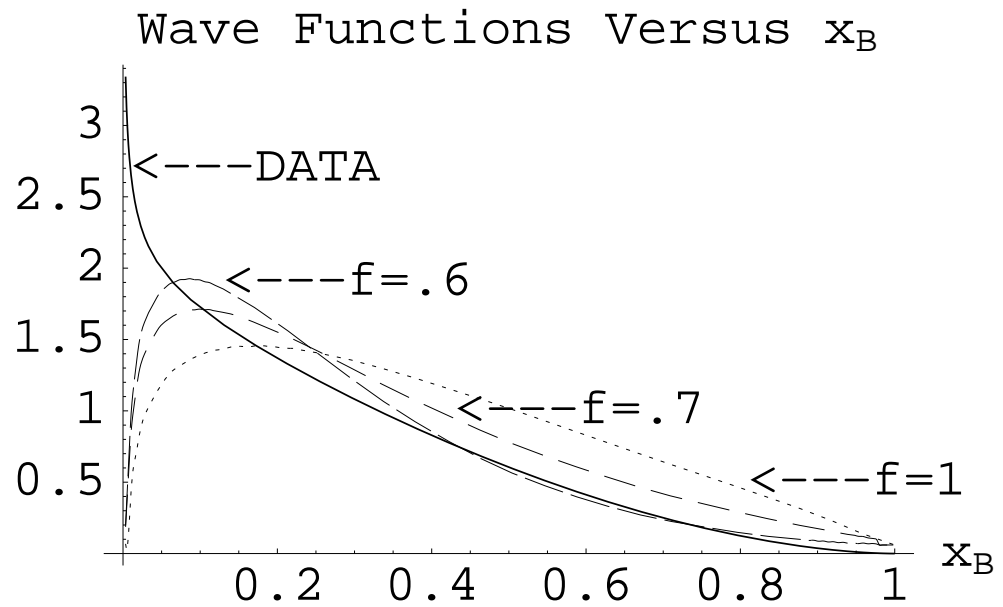

Comparison of parton wavefunctions $\sqrt{ } \phi(x)$ with the MRST global fit to data. 


\section{References}

[1] J. D. Bjorken and E. A. Paschos, Phys. Rev. 185 (1969) 1975 ; R. P. Feynman Photon Hadron Interactions Benjamin, Reading MA (1972).

[2] D. Gross and F. Wilczek Phys. Rev. Lett. 30 (1973) 1343 ;H. D. Politzer Phys. Rev. Lett. 30(1973) 1346 ; G. 't Hooft unpublished (1973).

[3] G. Sterman et al, Rev. Mod. Phys. 67(1995) 157.

[4] A. D. Martin, R. G. Roberts, W. J. Stirling and R. S. Thorne, hepph/9803445.

[5] M. Glück, E. Reya, Vogt, Z. Phys. C67(1995) 433.

[6] S. G. Rajeev, in it 1991 Summer School in High Energy Physics and Cosmology Vol.II, ed. E. Gava, K. Narain, S. Randjbar-Daemi, E. Sezgin and Q. Shafi, World Scientific, Singapore (1992); P. F. Bedaque, I. Horvath and S. G. Rajeev Mod. Phys. Lett. A7(1992) 3347; S. G. Rajeev, Int. J. Mod. Phys. A31(1994) 5583 ; O.T. Turgut and S. G. Rajeev, Comm. Math. Phys. $192(1998) 493$.

[7] S. J. Brodsky hep-ph/9807212.

[8] W. Hackbusch, Integral Equations: Theory and Numerical Treatment Birkhauser Verlag, Basel Switzerland (1995); P. J. Davis and P. Rabinowitz, Methods of Numerical Integration Academic Press, Orlando Florida (1984).

[9] T. H. R. Skyrme, Proc. Roy. Soc. A260 (1961) 127; Nucl. Phys. 31(1962) 556 ; J. Math. Phys. 12(1971) 1735 ; A.P. Balachandran, V.P.Nair, S.G.Rajeev and A.Stern, Phys. Rev. Lett. 49 (1982) 1124; G.Adkins, C.Nappi and E.Witten, Nucl. Phys B228(1983) 552 .

[10] G. 't Hooft, Nucl. Phys. B75(1974) 461.

[11] E. Witten, Nucl. Phys. B160 (1979) 57. 
[12] W. Kwong, J. Rosner and C. Quigg, Ann. Rev. Nucl. Part. Sci. 37(1987) 325. 